November 23, 2018

\title{
Parametric statistics of zeros of Husimi representations of quantum chaotic eigenstates and random polynomials
}

\author{
Tomaž Prosen \\ Physics Department, Faculty of Mathematics and Physics, \\ University of Ljubljana, Jadranska 19, 1000 Ljubljana, Slovenia
}

\begin{abstract}
Local parametric statistics of zeros of Husimi representations of quantum eigenstates are introduced. It is conjectured that for a classically fully chaotic systems one should use the model of parametric statistics of complex roots of Gaussian random polynomials which is exactly solvable as demonstrated below. For example, the velocities (derivatives of zeros of Husimi function with respect to an external parameter) are predicted to obey a universal (non-Maxwellian) distribution

$$
\frac{d \mathcal{P}(v)}{d v^{2}}=\frac{2}{\pi \sigma^{2}}\left(1+|v|^{2} / \sigma^{2}\right)^{-3},
$$

where $\sigma^{2}$ is the mean square velocity. The conjecture is demonstrated numerically in a generic chaotic system with two degrees of freedom. Dynamical formulation of the "zero-flow" in terms of an integrable many-body dynamical system is given as well.
\end{abstract}

\section{Introduction}

The intense research in the so-called quantum chaology has produced many different signatures of classical chaos in the corresponding quantum Hamiltonian systems. It has been found that energy spectra and eigenstates of classically fully chaotic quantum systems have universal statistical properties which can be described by stochastic models with no free parameters, like random matrix theory (RMT).

In the Bargmann (or Husimi) representation, eigenstates of quantum systems can be uniquely represented in terms of complex analytic functions in phase space variables. Quite recently, it has been proposed [7] that in case of 1-dim systems (or Poincaré surface

\footnotetext{
${ }^{1}$ e-mail: prosen@fiz.uni-lj.si
} 
of section reductions of 2-dim systems [10]), where phase space is two dimensional, and one has only one complex variable $z=q+i p$, any eigenstate can be uniquely represented by the collection of (complex) zeros of its Bargmann or Husimi representation. This has been called the stellar representation. It has been found [10 that the structure of zeros of Husimi representation of an eigenstate is reminiscent of the structure of classical phase space: (i) in classically fully chaotic systems the zeros tend to spread uniformly over the whole classically allowed region of phase space [7], (ii) in classically integrable systems the zeros lie on 1-dim. anti-Stokes curves [7], (iii) while in generic mixed systems a part of zeros uniformly cover chaotic components of classical phase space, zeros in regular islands lie along 1-dim. classically invariant curves - tori, while substantial part of zeros lie on 1-dim. classically noninvariant (generalized anti-Stokes) curves.

In the following we shall be interested only in the case of classically fully chaotic systems. Let us assume that the Hamiltonian of our chaotic system can be statistically described by Gaussian orthogonal/unitary (in the presence/absence of anti-unitary symmetry) random matrix in a generic basis. If we choose the harmonic basis then the Bargmann representation of an arbitrary eigenstate is given by the entire function

$$
f(z)=\sum_{n=0}^{\infty} \frac{c_{n}}{\sqrt{n !}} z^{n},
$$

and the Husimi representation in appropriately scaled units is $|f(z)|^{2} e^{-|z|^{2}}$, where $z=$ $q+i p . c_{n}$ are the coefficients of the expansion of that eigenstate in the harmonic basis, which are, by assumption and hence according to RMT, real/complex Gaussian (pseudo) random variables. Ideally, as RMT predicts, there should be no correlations among them $\left\langle c_{n} c_{m}^{*}\right\rangle=\delta_{n m}$, although we believe that for nonzero shortrange correlations among the coefficients $c_{n}$ our general conclusions are still valid. Since Taylor expansion of $f(z)$ around arbitrary point $z_{0}$ is convergent one may define and study Gaussian random polynomials of order $N$ and the statistical properties of their roots and in the end, if neccesary, take the 'thermodynamic' limit $N \rightarrow \infty$ (in a sense of increasing number of zeros - quasi-particles) of Gaussian random holomorphic functions (11). The limit $N \rightarrow \infty$ is in fact compatible with a semiclassical limit $\hbar \rightarrow 0$ since the order $N$ of a Taylor polynomial aproximating a Bargmann function (and its zeros) should be at least equal to the number of basis states covering the classically accessible area $A$ of phase space (or surface of section), $N>A /(2 \pi \hbar)$, while higher coefficients $a_{N+n}, n>0$, vanish rapidly with increasing $n$. One can also consider cases of different geometry, for example of kicked spin $j$ systems, where quantum (eigen)states can be exactly represented in terms of the so-called $\mathrm{SU}(2)$ polynomials of finite order $2 j$.

It has been shown recently by Hannay [5] for the case of complex coefficients and supplemented by Prosen [12] for the case of real coefficients that the statistics of zeros of Gaussian random polynomials are exactly solvable and all k-point correlation functions can be given in terms of simple analytical formulae. It has been demonstrated [6, 11] that the results obtained by random polynomials indeed reproduce the statistics of zeros of Husimi representations of chaotic eigenstates.

It is the aim of this paper to introduce the local parametric statistics of zeros of Husimi representations of the quantum eigenstates. Let us take a family of Hamiltonian systems which smoothly depend upon an external parameter $\lambda$. Then the Bargmann representation of a given eigenstate $f(z, \lambda)$ and its zeros $z_{n}(\lambda)$ are also smooth functions of the 
parameter $\lambda$. Therefore we can introduce the velocities $v_{n}$ as derivatives of zeros $z_{n}$ with respect to an external parameter $\lambda$

$$
v_{n}(\lambda)=\frac{d}{d \lambda} z_{n}(\lambda)=-\frac{\partial_{\lambda} f\left(z_{n}, \lambda\right)}{\partial_{z} f\left(z_{n}, \lambda\right)}
$$

Then we define a parametric k-point correlation function of $k$ zeros $\boldsymbol{z}=\left(z_{1}, \ldots, z_{k}\right)$ and $k$ velocities $\boldsymbol{v}=\left(v_{1}, \ldots, v_{k}\right)$ as

$$
\tilde{\rho}_{k}(\boldsymbol{z}, \boldsymbol{v})=\left\langle\delta^{2 k}\left(\boldsymbol{z}-\boldsymbol{z}^{\prime}\right) \delta^{2 k}\left(\boldsymbol{v}-\boldsymbol{v}^{\prime}\right)\right\rangle_{\boldsymbol{z}^{\prime}, \boldsymbol{v}^{\prime}}
$$

where \langle\rangle $\boldsymbol{z}^{\prime}, \boldsymbol{v}^{\prime}$ represents an (ensemble) average over all k-tuples of zeros $\boldsymbol{z}^{\prime}$ and corresponding velocities $\boldsymbol{v}^{\prime}$ of a given state (or ensemble of states). In other words, $\tilde{\rho}_{k}(\boldsymbol{z}, \boldsymbol{v}) d^{2 k} \boldsymbol{z} d^{2 k} \boldsymbol{v}$ is a probability to find a k-tuple of zeros and corresponding velocities in a small $4 k$-cube of volume $d^{2 k} \boldsymbol{z} d^{2 k} \boldsymbol{v}$ around point $(\boldsymbol{z}, \boldsymbol{v})$. Integrating out the velocities one should obtain the usual $k$-point correlation function $\rho_{k}(\boldsymbol{z})$ of zeros only [5, 12]

$$
\rho(\boldsymbol{z})=\int d^{2 k} \boldsymbol{v} \tilde{\rho}(\boldsymbol{z}, \boldsymbol{v}) .
$$

We show below, in section 2, that this parametric correlation functions can be explicitly calculated for Gaussian ensembles of parameter-dependent random polynomials with either complex or real coefficients. In section 3, we shall verify our conjecture that the obtained result on parametric statistics of roots of statistical ensembles of random polynomials may be applied to quantum chaotic systems by presenting some numerical results obtained in a generic chaotic system, namely 2-dim semi-separable oscillator [9, 10]. In section 4 , we write a closed system of 'equations of motion' for the zero-flow $z_{n}(\lambda)$ for the simplest, linear parametric dependence of the coefficients $a_{n}$, and stress the integrability of the underlying dynamical system.

\section{Parametric statistics of roots of Gaussian random polynomials}

\subsection{Complex coefficients}

In this section we study parametric statistics of statistical ensembles of Gaussian random polynomials. In the first subsection we are dealing with the case of complex coefficients while slightly more complicated case of real coefficients will be dealt with in the next subsection. Let us write a random polynomial of order $N$ in a form

$$
f(z, \lambda)=\sum_{n=0}^{N} a_{n}(\lambda) z^{n}
$$

where the coefficients $a_{n}(\lambda)$ (for a fixed realization) may depend smoothly on an external parameter $\lambda$. We will need only first derivatives with respect to $\lambda$ so the parametric statistical Gaussian ensemble of random polynomials is completely specified by fixing $\lambda$ and saying that $a_{n}$ and $\partial_{\lambda} a_{n}$ are complex Gaussian random variables with prescribed covariances

$$
\left\langle a_{n} a_{m}^{*}\right\rangle, \quad\left\langle a_{n} \partial_{\lambda} a_{m}^{*}\right\rangle, \quad\left\langle\partial_{\lambda} a_{n} \partial_{\lambda} a_{m}^{*}\right\rangle
$$


which need not be further specified for the statement of the general result. Fixing the two $k$-tuples of complex numbers $\boldsymbol{z}$ and $\boldsymbol{v}$, we define the $3 k$ linear combinations of $2(N+1)$ Gaussian random variables $a_{n}, \partial_{\lambda} a_{n}, n=0, \ldots, N$

$$
\begin{aligned}
& f_{j}=f\left(z_{j}, \lambda\right)=\sum_{n=0}^{N} a_{n} z_{j}^{n}, \\
& f_{j}^{\prime}=\partial_{z} f\left(z_{j}, \lambda\right)=\sum_{n=0}^{N-1}(n+1) a_{n+1} z_{j}^{n}, \\
& \tilde{f}_{j}=\frac{d}{d \lambda} f\left(z_{j}, \lambda\right)=\bar{f}_{j}+v_{j} f_{j}^{\prime}, \quad \bar{f}_{j}=\partial_{\lambda} f\left(z_{j}, \lambda\right)=\sum_{n=0}^{N} \partial_{\lambda} a_{n} z_{j}^{n}
\end{aligned}
$$

which are again Gaussian random variables. The joint probability distribution of $3 k$ random variables $\boldsymbol{\xi}=\left(\boldsymbol{f}, \boldsymbol{f}^{\prime}, \tilde{\boldsymbol{f}}\right)$ can be therefore written as

$$
P\left(\boldsymbol{f}, \boldsymbol{f}^{\prime}, \tilde{\boldsymbol{f}}\right)=\frac{1}{\pi^{3 k} \operatorname{det} \tilde{\mathrm{M}}} \exp \left(-\boldsymbol{\xi}^{*} \cdot \tilde{\mathrm{M}}^{-1} \boldsymbol{\xi}\right)
$$

where $\tilde{\mathrm{M}}=\tilde{\mathrm{M}}(\boldsymbol{z}, \boldsymbol{v})$ is $3 k \times 3 k$ Hermitian covariance matrix which is written in a block form as

$$
\begin{aligned}
& \tilde{M}=\left\langle\boldsymbol{\xi} \otimes \boldsymbol{\xi}^{*}\right\rangle=\left(\begin{array}{ccc}
\mathrm{A} & \mathrm{B} & \tilde{\mathrm{D}} \\
\mathrm{B}^{\dagger} & \mathrm{C} & \tilde{\mathrm{E}} \\
\tilde{\mathrm{D}}^{\dagger} & \tilde{\mathrm{E}}^{\dagger} & \tilde{\mathrm{F}}
\end{array}\right) \\
& A_{j l}=\left\langle f_{j} f_{l}^{*}\right\rangle, \quad B_{j l}=\left\langle f_{j} f_{l}^{\prime *}\right\rangle, \quad C_{j l}=\left\langle f_{j}^{\prime} f_{l}^{\prime *}\right\rangle \text {, } \\
& \tilde{D}_{j l}=\left\langle f_{j} \tilde{f}_{l}^{*}\right\rangle, \quad \tilde{E}_{j l}=\left\langle f_{j}^{\prime} \tilde{f}_{l}^{*}\right\rangle, \quad \tilde{F}_{j l}=\left\langle\tilde{f}_{j} \tilde{f}_{l}^{*}\right\rangle .
\end{aligned}
$$

Note that tilded symbols are used to denote matrices (or vectors or scalars) which explicitly depend on the parametric velocities $\boldsymbol{v}$. $k$-tuple $\boldsymbol{z}$ are the zeros if $\boldsymbol{f}=0$, and $k$-tuple $\boldsymbol{v}$ are the velocities if in addition $\tilde{\boldsymbol{f}} \equiv(d / d \lambda) \boldsymbol{f}=0$. So the parametric $k$-point correlation function (3) can be written as a linear transformation of a joint distribution $P(\boldsymbol{\xi})$

$$
\begin{aligned}
\tilde{\rho}(\boldsymbol{z}, \boldsymbol{v}) & =\int d^{2 k} \boldsymbol{f}^{\prime} \frac{\partial(\boldsymbol{f}, \tilde{\boldsymbol{f}})}{\partial(\boldsymbol{z}, \boldsymbol{v})} P\left(\mathbf{0}, \boldsymbol{f}^{\prime}, \mathbf{0}\right) \\
& =\frac{1}{\pi^{3 k} \operatorname{det} \mathrm{M}} \int \prod_{j=1}^{k} d^{2} f_{j}^{\prime}\left|f_{j}^{\prime}\right|^{4} \exp \left(-\boldsymbol{f}^{\prime *} \cdot \tilde{\mathrm{L}}^{-1} \boldsymbol{f}^{\prime}\right)
\end{aligned}
$$

where $\partial(\boldsymbol{f}, \tilde{\boldsymbol{f}}) / \partial(\boldsymbol{z}, \boldsymbol{v})=\prod_{j=1}^{k}\left|f_{j}^{\prime}\right|^{4}$ is the Jacobian of the mapping $(\boldsymbol{z}, \boldsymbol{v}) \rightarrow(\boldsymbol{f}, \tilde{\boldsymbol{f}})$, and

$$
\tilde{\mathrm{L}}=\mathrm{C}-\mathrm{B}^{\dagger} \mathrm{A}^{-1} \mathrm{~B}-\left(\tilde{\mathrm{E}}-\mathrm{B}^{\dagger} \mathrm{A}^{-1} \tilde{\mathrm{D}}\right)\left(\tilde{\mathrm{F}}-\tilde{\mathrm{D}}^{\dagger} \mathrm{A}^{-1} \tilde{\mathrm{D}}\right)^{-1}\left(\tilde{\mathrm{E}}^{\dagger}-\tilde{\mathrm{D}}^{\dagger} \mathrm{A}^{-1} \mathrm{~B}\right)
$$

is the central $k \times k$ block of the inverse of covariance matrix, $\tilde{\mathrm{M}}^{-1}$. The dependence on positions of zeros $\boldsymbol{z}$ and velocities $\boldsymbol{v}$ is digged in the definitions of the matrices (10, 11, 12) In general, the dependence on velocities can be made explicit in the following way. Writing a diagonal velocity matrix as $\mathrm{V}=\operatorname{diag}\left\{v_{j}, j=1 \ldots k\right\}$ and using a definition (8) one can observe that the covariance matrices $\tilde{\mathrm{D}}, \tilde{\mathrm{E}}, \tilde{\mathrm{F}}$ have a simple velocity dependence which can 
be written in terms of their velocity-independent counterparts $\mathrm{D}, \mathrm{E}, \mathrm{F}$

$$
\begin{aligned}
\tilde{\mathrm{D}} & =\mathrm{D}+\mathrm{BV}^{\dagger}, \quad D_{j l}=\left\langle f_{j} \bar{f}_{l}^{*}\right\rangle, \\
\tilde{\mathrm{E}} & =\mathrm{E}+\mathrm{CV}^{\dagger}, \quad E_{j l}=\left\langle f_{j}^{\prime} \bar{f}_{l}^{*}\right\rangle, \\
\tilde{\mathrm{F}} & =\mathrm{F}+\mathrm{E}^{\dagger} \mathrm{V}^{\dagger}+\mathrm{VE}+\mathrm{VCV}^{\dagger}, \quad F_{j l}=\left\langle\bar{f}_{j} \bar{f}_{l}^{*}\right\rangle
\end{aligned}
$$

This relations can be used to prove that the determinant of the covariance matrix does not depend on velocities

$$
\operatorname{det} \tilde{\mathrm{M}}=\operatorname{det} \mathrm{M}
$$

where $3 k \times 3 k$ matrix $\mathrm{M}$ is obtained from $\tilde{\mathrm{M}}$ by replacing the blocks $\tilde{\mathrm{D}}, \tilde{\mathrm{E}}, \tilde{\mathrm{F}}$ by $\mathrm{D}, \mathrm{E}, \mathrm{F}$. Using some elementary algebra one can rewrite the matrix $\tilde{L}$ in the form which makes velocity dependence explicit

$$
\tilde{\mathrm{L}}^{-1}=\mathrm{G}^{-1}+\left(\mathrm{V}^{\dagger}+\mathrm{G}^{-1} \mathrm{~K}\right)\left(\mathrm{H}-\mathrm{K}^{\dagger} \mathrm{G}^{-1} \mathrm{~K}\right)^{-1}\left(\mathrm{~V}+\mathrm{K}^{\dagger} \mathrm{G}^{-1}\right)
$$

where we have introduced the matrices

$$
\begin{aligned}
& \mathrm{G}=\mathrm{C}-\mathrm{B}^{\dagger} \mathrm{A}^{-1} \mathrm{~B}=\mathrm{G}^{\dagger}, \\
& \mathrm{H}=\mathrm{F}-\mathrm{D}^{\dagger} \mathrm{A}^{-1} \mathrm{D}=\mathrm{H}^{\dagger}, \\
& \mathrm{K}=\mathrm{E}-\mathrm{B}^{\dagger} \mathrm{A}^{-1} \mathrm{D} .
\end{aligned}
$$

The representation of parametric correlations $\tilde{\rho}_{k}$ in terms of moments of a Gaussian (14) is very convenient since it may be explicitly evaluated as the sum of all possible pairwise contractions of integration variables $f_{j}^{\prime}$ (Wick theorem) and expressed in a compact form following an approach of Hannay [5]

$$
\tilde{\rho}_{k}(\boldsymbol{z}, \boldsymbol{v})=\frac{\operatorname{det} \tilde{\mathrm{L}}}{\pi^{2 k} \operatorname{det} \mathrm{M}} \operatorname{per}\left(\begin{array}{cc}
\tilde{\mathrm{L}} & \tilde{\mathrm{L}} \\
\tilde{\mathrm{L}} & \tilde{\mathrm{L}}
\end{array}\right)
$$

where permanent of a square matrix per $S=\sum_{p} \prod_{j} S_{j p_{j}}$ is a symmetric analog of a determinant det $S=\sum_{p}(-)^{p} \prod_{j} S_{j p_{j}}$ where $p$ are permutations with signatures $(-)^{p}$. Integrating out the velocities, which can be done by putting expression (18) into eq. (14) and evaluating the inner Gaussian integrals in terms of new variables $u_{j}=v_{j} f_{j}^{\prime}$, one obtains the $k$-point correlation function of Hannay [ [0]

$$
\rho_{k}(\boldsymbol{z})=\frac{\operatorname{per} \mathrm{G}}{\pi^{k} \operatorname{det} \mathrm{A}} .
$$

The formula (20) is a general result on parametric statistics of Gaussian random polynomials with complex coefficients. Its important feature is purely algebraic dependence on velocities in contradistinction with e.g. parametric energy level statistics (see e.g. [4], chapter 6) where velocities have Maxwellian distribution since the Hamiltonian of the energy level flow can be clearly written as the sum of the usual kinetic and potential part.

In the important special case where (the coefficients of) the random polynomial and its parametric derivative are statistically uncorrelated

$$
\left\langle a_{n} \partial_{\lambda} a_{m}\right\rangle=0
$$


we obtain parametric correlation functions which are invariant under the change of sign of velocities $\tilde{\rho}_{k}(\boldsymbol{z},-\boldsymbol{v})=\tilde{\rho}_{k}(\boldsymbol{z}, \boldsymbol{v})$ since $\mathrm{E}=\mathrm{D}=\mathrm{K}=0$ and therefore

$$
\tilde{\mathrm{L}}^{-1}=\mathrm{G}^{-1}+\mathrm{V}^{\dagger} \mathrm{F}^{-1} \mathrm{~V}
$$

In other words, the average velocity (and all its odd moments) is zero $\langle\boldsymbol{v}\rangle=0$. In this case, the determinant of $3 k \times 3 k$ covariance matrix has also a simple factorization in terms of $k \times k$ matrices

$$
\operatorname{det} \mathrm{M}=\operatorname{det} \mathrm{A} \operatorname{det} \mathrm{G} \operatorname{det} \mathrm{F} \text {. }
$$

Since the 2-point parametric correlation function depends on 4-complex arguments it may be useful to define also the 2-point velocity-moments of the parametric correlation functions

$$
\left\langle v_{1}^{k} v_{2}^{l} v_{1}^{* m} v_{2}^{* n}\right\rangle=\frac{1}{\rho_{2}\left(z_{1}, z_{2}\right)} \int d^{2} v_{1} d^{2} v_{2} v_{1}^{k} v_{2}^{l} v_{1}^{* m} v_{2}^{* n} \tilde{\rho}_{2}\left(z_{1}, z_{2}, v_{1}, v_{2}\right)
$$

which are different from zero only if $k+l=m+n$ and finite if $k+l+m+n \leq 4$. The nontrivial velocity-moments, which still depend on the positions of two zeros $z_{1}$ and $z_{2}$, can be calculated using the Wick theorem from the representation (14). Let us quote the results for the driftless case (22)

$$
\begin{aligned}
\left\langle v_{1} v_{2}^{*}\right\rangle & =G_{21} F_{12} /\left(G_{11} G_{22}+G_{12} G_{21}\right), \\
\left\langle\left|v_{1}\right|^{2}\left|v_{2}\right|^{2}\right\rangle & =\left(F_{11} F_{22}+F_{12} F_{21}\right) /\left(G_{11} G_{22}+G_{12} G_{21}\right), \\
\left\langle v_{1}^{2} v_{2}^{* 2}\right\rangle & =2 F_{12}^{2} /\left(G_{11} G_{22}+G_{12} G_{21}\right) .
\end{aligned}
$$

So far the correlations between the coefficients of random polynomials $a_{n}, \partial_{\lambda} a_{n}$ have been completely arbitrary! Now we specialize to the case where coefficients of random polynomials are $\delta$-correlated

$$
\left\langle a_{n} a_{m}^{*}\right\rangle=\delta_{m n} b_{n}, \quad\left\langle\partial_{\lambda} a_{n} \partial_{\lambda} a_{m}^{*}\right\rangle=\delta_{m n} \bar{b}_{n}
$$

where the variances $b_{n}, \bar{b}_{n}$ are still arbitrary. Introducing two polynomials

$$
g(s)=\sum_{n=0}^{N} b_{n} s^{n}, \quad \bar{g}(s)=\sum_{n=0}^{N} \bar{b}_{n} s^{n}
$$

the relevant matrices can be expressed as

$$
\begin{aligned}
A_{j l}(\boldsymbol{z}) & =g\left(z_{j} z_{l}^{*}\right) \\
B_{j l}(\boldsymbol{z}) & =z_{j} g^{\prime}\left(z_{j} z_{l}^{*}\right) \\
C_{j l}(\boldsymbol{z}) & =g^{\prime}\left(z_{j} z_{l}^{*}\right)+z_{j} z_{l}^{*} g^{\prime \prime}\left(z_{j} z_{l}^{*}\right) \\
F_{j l}(\boldsymbol{z}) & =\bar{g}\left(z_{j} z_{l}^{*}\right) .
\end{aligned}
$$

Putting $k=1,1$-point parametric statistics can be explicitly written in an elegant factorized form

$$
\tilde{\rho}_{1}(z, v)=\rho_{1}(z) \frac{\tilde{\nu}(v / \sigma(z))}{\sigma^{2}(z)}
$$


where

$$
\rho_{1}(z)=\left.\frac{1}{\pi} \frac{d}{d s} s \frac{d}{d s} \log g(s)\right|_{s=|z|^{2}}
$$

is a general density of zeros as can be deduced from [5] and

$$
\tilde{\nu}(v)=\frac{2}{\pi}\left(1+|v|^{2}\right)^{-3}
$$

is an universal velocity distribution normalized to a unit mean square and $\sigma^{2}(z)=\left\langle|v|^{2}\right\rangle$ is a mean square velocity which is inversely proportional to the density of zeros

$$
\sigma^{2}(z)=\frac{\bar{g}\left(|z|^{2}\right)}{g\left(|z|^{2}\right)} \frac{1}{\pi \rho_{1}(z)} .
$$

So, the theory of random polynomials predicts a universal form of a velocity distribution (35) when it is locally rescaled to a unit mean square local velocity.

In case of eigenstates of RMT in the Bargmann representation one has $b_{n}=1 / n !, \bar{b}_{n}=$ $\sigma^{2} / n !$ and $N \rightarrow \infty$, so

$$
g(s)=\exp (s), \quad \bar{g}(s)=\sigma^{2} \exp (s)
$$

and therefore the density distribution and the local mean square velocity are constant, $\rho_{1}(z)=1 / \pi,\left\langle|v|^{2}\right\rangle=\sigma^{2}$, so one has

$$
\tilde{\rho}_{1}(z, v)=\frac{2}{\pi^{2} \sigma^{2}}\left(1+|v|^{2} / \sigma^{2}\right)^{-3}
$$

In this probably the most important particular case we are also able to give some details of 2-point parametric correlation function $\tilde{\rho}_{2}\left(z_{1}, z_{2}, v_{1}, v_{2}\right)$ which is only a function of the 4 real quantities instead of 8: distance between roots $\left|z_{2}-z_{1}\right|$, magnitudes of the velocities $u_{1}=\left|v_{1}\right|, u_{2}=\left|v_{2}\right|$ and the angle between velocities $\phi=\arccos \left(\operatorname{Re} v_{1} v_{2}^{*} /\left|v_{1} v_{2}\right|\right)$. Writing $s=\left|z_{2}-z_{1}\right|^{2}$ and fixing the velocity scale by putting $\sigma=1$ we may express 2 -point parametric correlations as

$$
\begin{aligned}
\tilde{\rho}_{2} & =\frac{4\left(e^{s}-1\right)^{5} \alpha^{5}\left(\beta^{2}+\gamma^{2}+4 \beta \gamma\right)}{\pi^{2}(\beta-\gamma)^{5}} \\
\alpha & =e^{2 s}-\left(s^{2}+2\right) e^{s}+1 \\
\beta & =e^{s}\left(\left(e^{s}-1\right)\left(e^{s}-1-s\right)+\alpha u_{1}^{2}\right)\left(\left(e^{s}-1\right)\left(e^{s}-1-s\right)+\alpha u_{2}^{2}\right) \\
\gamma & =e^{2 s}\left(e^{s}-1\right)^{2}\left(e^{-s}-1+s\right)^{2}+\alpha^{2} u_{1}^{2} u_{2}^{2}-2 \alpha e^{s}\left(e^{s}-1\right)\left(e^{-s}-1+t\right) u_{1} u_{2} \cos (\phi)
\end{aligned}
$$

Since this expression is quite complicated it is worthwhile to study its asymptotics for large and small distances $\sqrt{s}$ between the roots. The first two nonzero terms of the small $s$ expansion are

$$
\tilde{\rho}_{2}=\frac{48}{\pi^{4}\left(2+\left|v_{1}+v_{2}\right|^{2}\right)^{5}} s^{2}+\frac{8\left(\left|v_{1}^{2}-v_{2}^{2}\right|^{2}-8\left|v_{1}-v_{2}\right|^{2}\right)}{\pi^{4}\left(2+\left|v_{1}+v_{2}\right|^{2}\right)^{6}} s^{3}+\mathcal{O}\left(s^{4}\right) .
$$

For large $s$ asymptotics in the leading term, as we expect, the two velocities are uncorrelated, while we give also the next term of an expansion in powers of $e^{-s}$

$$
\begin{aligned}
\tilde{\rho}_{2} & =\frac{4}{\pi^{4}}\left[\frac{1+6 e^{-s}}{\left(1+\left|v_{1}\right|^{2}\right)^{3}\left(1+\left|v_{2}\right|^{2}\right)^{3}}\right. \\
& +\frac{s^{2}\left(\left|v_{1}\right|^{2}-2\right)\left(\left|v_{2}\right|^{2}-2\right)+12 s\left(\left|v_{1}\right|^{2}+\left|v_{2}\right|^{2}-1\right)-9 s\left|v_{1}+v_{2}\right|^{2}-9\left|v_{1}-v_{2}\right|^{2}}{\left(1+\left|v_{1}\right|^{2}\right)^{4}\left(1+\left|v_{2}\right|^{2}\right)^{4}} e^{-s} \\
& \left.+\mathcal{O}\left(e^{-2 s}\right)\right]
\end{aligned}
$$


Note that the small $s$ expansion (40) should be understood strictly pointwise, while it is not termwise integrable with respect to velocities $v_{1}$ and $v_{2}$ as should be the case for the entire 2-point parametric correlation function $\tilde{\rho}_{2}$ (3). It is useful to give also the velocity-moments (23) which in this case depend only on the distance between zeros $\sqrt{s}$ and probably still contain a lot of information about parametric 2-point statistics

$$
\begin{aligned}
\left\langle v_{1} v_{2}^{*}\right\rangle & =-\sigma^{2} e^{s}\left(e^{s}-1\right)\left(e^{-s}-1+s\right) / \omega, \\
\left\langle\left|v_{1}\right|^{2}\left|v_{2}\right|^{2}\right\rangle & =\sigma^{4}\left(e^{s}+1\right)\left(e^{s}-1\right)^{2} / \omega \\
\left\langle v_{1}^{2} v_{2}^{* 2}\right\rangle & =2 \sigma^{4}\left(e^{s}-1\right)^{3} / \omega \\
\omega & =e^{s}\left(e^{s}-1-s\right)^{2}+e^{2 s}\left(e^{-s}-1+s\right)^{2} .
\end{aligned}
$$

\section{$2.2 \quad$ Real coefficients}

In this subsection we discuss the case of parametric statistics of the Gaussian random polynomials with real coefficients, i.e. $a_{n}$ and $\partial_{\lambda} a_{n}$ are real Gaussian random variables with prescribed covariances

$$
\left\langle a_{n} a_{m}\right\rangle, \quad\left\langle a_{n} \partial_{\lambda} a_{m}\right\rangle, \quad\left\langle\partial_{\lambda} a_{n} \partial_{\lambda} a_{m}\right\rangle .
$$

Fixing the two k-tuples of complex numbers $\boldsymbol{z}$ and $\boldsymbol{v}$ we define $6 k$ real random variables $\operatorname{Re} f_{j}, \operatorname{Im} f_{j}, \operatorname{Re} f_{j}^{\prime}, \operatorname{Im} f_{j}^{\prime}, \operatorname{Re} \tilde{f}_{j}, \operatorname{Im} \tilde{f}_{j}$, or equivalently, a vector of $3 \times 2 k$ variables $\boldsymbol{\xi}=$ $\left(\boldsymbol{f}, \boldsymbol{f}^{\prime}, \tilde{\boldsymbol{f}}\right)$ where $\boldsymbol{f}=\left(f_{1}, f_{1}^{*}, \ldots, f_{k}, f_{k}^{*}\right), \boldsymbol{f}^{\prime}=\left(f_{1}^{\prime}, f_{1}^{\prime *}, \ldots, f_{k}^{\prime}, f_{k}^{\prime *}\right), \tilde{\boldsymbol{f}}=\left(\tilde{f}_{1}, \tilde{f}_{1}^{*}, \ldots, \tilde{f}_{k}, \tilde{f}_{k}^{*}\right)$ The joint distribution of $\boldsymbol{\xi}$ is now again a (real) Gaussian with various blocks and their derivations of covariance matrices which are now $2 k \times 2 k$ matrices and are defined by the same formulae (10,11, 12, 16, 18, 19). Using straightforward approach which follows the previous subsection and the derivation of nonparametric statistics for real coefficients [12] one derives the general formula for the parametric $k$-point correlation function of zeros of Gaussian random polynomials with real coefficients

$$
\tilde{\rho}_{k}(\boldsymbol{z}, \boldsymbol{v})=\frac{1}{(2 \pi)^{2 k}} \sqrt{\frac{\operatorname{det} \tilde{\mathrm{L}}}{\operatorname{det} \mathrm{M}}} \operatorname{sper}\left(\begin{array}{ccccc}
\tilde{L}_{11} & \tilde{L}_{11} & \ldots & \tilde{L}_{12 k} & \tilde{L}_{12 k} \\
\tilde{L}_{11} & \tilde{L}_{11} & \ldots & \tilde{L}_{12 k} & \tilde{L}_{12 k} \\
\vdots & \vdots & \ddots & \vdots & \vdots \\
\tilde{L}_{2 k 1} & \tilde{L}_{2 k 1} & \ldots & \tilde{L}_{2 k 2 k} & \tilde{L}_{2 k 2 k} \\
\tilde{L}_{2 k 1} & \tilde{L}_{2 k 1} & \ldots & \tilde{L}_{2 k 2 k} & \tilde{L}_{2 k 2 k}
\end{array}\right)
$$

which reduces to the corresponding nonparametric $k$-point correlation function [12]

$$
\rho_{k}(\boldsymbol{z})=\frac{\text { sper } \mathrm{G}}{(2 \pi)^{k} \sqrt{\operatorname{det} \mathrm{A}}}
$$

after velocities are integrated out. Semi-permanent of a square $2 m \times 2 m$ matrix (introduced in [12]) is a homogeneous polynomial of order $m$ of the matrix elements

$$
\text { sper } \mathrm{S}=\sum_{\substack{j_{1}<\ldots<j_{m} \\ l_{1}<\ldots<l_{m}}}^{j_{n} \neq l_{n^{\prime}}} \sum_{p} \prod_{r=1}^{m} S_{j_{r}+m, l_{p_{r}}} .
$$

where $p$ runs over all $m$ ! permutations of $m$ indices $\{1 \ldots m\}$ and addition of indices $j_{r}+m$

should be understood modulo $2 m$. Note that $\operatorname{sper}\left(\left(\begin{array}{ll}1 & 1 \\ 1 & 1\end{array}\right) \otimes \tilde{\mathrm{L}}\right) \neq \operatorname{sper}\left(\tilde{\mathrm{L}} \otimes\left(\begin{array}{ll}1 & 1 \\ 1 & 1\end{array}\right)\right)$, 
whereas per $\left(\left(\begin{array}{ll}1 & 1 \\ 1 & 1\end{array}\right) \otimes \tilde{\mathrm{L}}\right)=\operatorname{per}\left(\tilde{\mathrm{L}} \otimes\left(\begin{array}{ll}1 & 1 \\ 1 & 1\end{array}\right)\right)$, so the $4 k \times 4 k$ matrix on the RHS of eq. (46) cannot be written as $\left(\begin{array}{cc}\tilde{\mathrm{L}} & \tilde{\mathrm{L}} \\ \tilde{\mathrm{L}} & \tilde{\mathrm{L}}\end{array}\right)$ like in the complex case (20).

Since the case of real coefficients is much more complicated than the case of complex coefficients we consider only the final and most important specialization, that is of harmonic random polynomials with real coefficients in the 'thermodynamic' limit $N \rightarrow \infty$, $\left\langle a_{n} a_{m}\right\rangle=\delta_{n m} / n !,\left\langle a_{n} \partial_{\lambda} a_{m}\right\rangle=0,\left\langle\partial_{\lambda} a_{n} \partial_{\lambda} a_{m}\right\rangle=\sigma^{2} \delta_{n m} / n !, g(s)=\exp (s), \bar{g}(s)=\sigma^{2} \exp (s)$. Then it is easy to see (like in the nonparametric case [12]) that the two cases of real and complex coefficients are different only when some of the zeros $z_{j}$ is close to the real (symmetry) axis, whereas in the opposite case, where all $z_{j}$ go away from the real axis, the parametric statistics for the case of real coefficients converge to parametric statistics for the complex coefficients. Explicit formulae for 1 and 2 point parametric correlation functions for this case are too lengthy to write out, we shall only give an asymptotic expansion for 1 -point function close to the real axis ( $\operatorname{small} y=\operatorname{Im} z$ )

$$
\tilde{\rho}_{1}(x+i y, u+i w)=\frac{48 \sigma y^{2}}{\pi^{2}\left(\sigma^{2}+2 u^{2}\right)^{3 / 2}}-\frac{8 \sigma\left(u^{4}+3 u^{2} w^{2}\right) y^{4}}{\pi^{2}\left(\sigma^{2}+2 u^{2}\right)^{7 / 2}}+\mathcal{O}\left(y^{6}\right)
$$

It is interesting to calculate the 'root mean square' (RMS) velocity which now depends on the distance from the real axis $y$

$$
\left\langle|v|^{2}\right\rangle=\sigma^{2} \frac{e^{4 y^{2}}-1}{e^{4 y^{2}}-1-4 y^{2}}
$$

The fact that RMS velocity has a singularity around $s=0,\left\langle|v|^{2}\right\rangle \propto s^{-2}$, is compatible with finding that the small $s$ expansion of the parametric density (49) is not termwise integrable with respect to the velocity.

\section{$3 \quad$ Numerical results}

The predictions of the theory of random polynomials have been verified on the parametric statistics of zeros of Husimi representation of eigenfunctions of a generic chaotic autonomous Hamiltonian system with two freedoms which depends on an external parameter. We have chosen a recently introduced semi-separable oscillator for which an extremely efficient quantization scheme exists based on a construction of an exact quantum Poincare mapping [9, 10]. The Hamiltonian of the system is the following

$$
H=-\frac{1}{2} \hbar^{2} \partial_{x}^{2}-\frac{1}{2} \hbar^{2} \partial_{y}^{2}+\frac{1}{2}(x-a \operatorname{sign}(y))^{2}
$$

where the configuration space is a strip $(x, y) \in(-\infty, \infty) \times\left[b_{\downarrow}, b_{\uparrow}\right]$ with hard walls and therefore Dirichlet boundary conditions for a wavefunction at $y=b_{\downarrow}, b_{\uparrow}$. The system is integrable for $a=0$ while for $a>0$ the potential becomes discontinuous and the system becomes increasingly chaotic with increasing $a$. It has been shown in 10 that the system is fully chaotic (with possible islands of stability being negligibly small) for the following values of parameters: $a=0.25, b_{\uparrow}=4, b_{\downarrow}=-11, E=0.5$. For these values and for $\hbar=$ 0.0003 we have calculated a stretch of 16 consecutive eigenstates with sequential quantum number (according to Thomas-Fermi rule) $\approx 17684000$. Then we have calculated a second 
set of eigenstates according to variation of parameters $a=0.25, b_{\uparrow}=4+\lambda, b_{\downarrow}=-11+\lambda$ with $\lambda=5 \cdot 10^{-7}$. For each of the eigenstates of the two sets we have calculated the Husimi representation on the surface of section $\left(x, p_{x}\right)$ [10], its zeros (only inside classically allowed (chaotic) region of phase space) and calculated the variations of zeros w.r.t. small variation of the parameter from $\lambda=0$ to $\lambda=5 \cdot 10^{-7}$. Since the variation of the external parameter $\delta \lambda=5 \cdot 10^{-7}$ was small enough, the identification of corresponding zeros has always been possible and the velocities were numerically well defined. Assuming the conjecture that the parametric statistics of zeros of Husimi representation of a chaotic eigenstate should be described by the theory of random polynomials, only two parameters remain which define the scales, namely the average density of zeros $\rho_{1}$ (which should be constant inside the classically allowed chaotic region of surface of section) and the root mean square velocity $\sigma=\sqrt{\left\langle|v|^{2}\right\rangle}$. The density or the number of inside zeros is roughly constant for our 16 states while RMS velocity $\sigma$, which is determined by the sensitivity of a given eigenstate with respect to the variation of external parameter $\lambda$, might exhibit substantial fluctuations. Therefore, if we want to merge numerical data of all eigenstates together in order to improve statistics, we should first rescale the data for each individual eigenstate to a unit RMS velocity.

In figure 1 we plot such numerical velocity distribution for our stretch of 16 eigenstates which contains velocities for $\approx 16 \times 1800=28800$ zeros (only the zeros whose distance from the boundary of classically allowed region of surface of section was larger than few mean spacings were taken into account). It is evident that the agreement with the prediction of theory of random polynomials (35) is statistically significant.

In order to compare significantly the numerical estimate of the parametric 2 -point (or higher) statistics with the prediction of the theory of random polynomials one needs a data of a higher statistical quality than those of our numerical experiment. This may be easier in some explicit one-dimensional quantum maps and we leave it as a challenge for a future work.

\section{Zero-flow as an integrable dynamical system}

In this section we show explicitly that the flow of zeros of a polynomial with respect to some external parameter $\lambda$ interpreted as a fictitious 'time' can be written in terms of a closed set of 'equations of motion'. We argue that the underlying dynamical system is completely integrable and construct explicitly the complete set of integrals of motion as functions of zeros and velocities. This construction should be considered as a polynomial analog of Dyson-Pechukas-Yukawa [8, 13] level dynamics of matrices.

Let us consider a polynomial of order $N$ whose dependence on the external parameter $\lambda$ is linear

$$
\begin{aligned}
f(z, \lambda) & =\prod_{j=1}^{N}\left(z-z_{j}(\lambda)\right)=f_{0}(z)+\lambda f_{1}(z), \\
f_{0}(z) & =\sum_{n=0}^{N} a_{n}^{0} z^{n}, \quad f_{1}(z)=\sum_{n=0}^{N} a_{n}^{1} z^{n} .
\end{aligned}
$$

where without essential loss of generality we have assumed that

$$
a_{N}^{0}=1, \quad a_{N}^{1}=0 .
$$


Writing the velocities $v_{j}=(d / d \lambda) z_{j}=-f_{1}\left(z_{j}\right) / \prod_{l \neq j}\left(z_{j}-z_{l}\right)$, differentiating with respect to $\lambda$ again and using Lagrange interpolation formula for $f_{1}\left(z_{j}\right)$ and $f_{1}^{\prime}\left(z_{j}\right)$ one arrives to the simple closed set of equations for $2 N$ complex (or $4 N$ real) dynamical variables, zeros $z_{j}$ and velocities $v_{j}, j=1, \ldots, N$

$$
\begin{aligned}
\frac{d}{d \lambda} z_{j} & =v_{j} \\
\frac{d}{d \lambda} v_{j} & =2 \sum_{k \neq j} \frac{v_{j} v_{k}}{z_{j}-z_{k}}
\end{aligned}
$$

From the construction it is obvious that the underlying dynamical system should be completely integrable. Writing the polynomial

$$
f(z, \lambda)=\prod_{j=1}^{N}\left(z-z_{j}\right)=\sum_{n=0}^{N}(-1)^{n} c_{n}\left(z_{1}, \ldots, z_{N}\right) z^{N-n}
$$

in terms of fully symmetric homogeneous functions

$$
c_{n}\left(z_{1}, \ldots, z_{N}\right)=\sum_{\mathcal{A} \subset\{1, \ldots, N\}}^{|\mathcal{A}|=n} \prod_{j \in \mathcal{A}} z_{j}
$$

one easily sees that their derivatives

$$
I_{n}\left(z_{1}, \ldots, z_{N}, v_{1}, \ldots, v_{N}\right)=\frac{d}{d \lambda} c_{n}\left(z_{1}, \ldots, z_{N}\right)=\sum_{j=1}^{N} v_{j} \partial_{z_{j}} c_{n}
$$

are just equal to the coefficients of the perturbation polynomial (see eqs. (52,55)

$$
I_{n}=(-1)^{n} a_{N-n}^{1}
$$

and therefore independent nontrivial (complex) constants of motion, for $n=1, \ldots N$. It seems more difficult to give a simple Lagrangian or Hamiltonian formulation of the equations (54). Since the system is completely integrable its Lagrange function is not unique. However, it is easy to show that eqs. (54) are completely equivalent to Euler-Lagrange equations for any Lagrange function $L\left(z_{1}, \ldots, z_{N}, v_{1}, \ldots, z_{N}\right)$ which can be written in terms of a symmetric (real) and nondegenerate holomorphic function of constants of motion and their complex conjugates

$$
\begin{aligned}
L & =F\left(I_{1}, \ldots, I_{N}, I_{1}^{*}, \ldots, I_{N}^{*}\right), \\
F\left(I_{1}, \ldots, I_{N}, I_{1}^{*}, \ldots, I_{N}^{*}\right) & =F\left(I_{1}^{*}, \ldots, I_{N}^{*}, I_{1}, \ldots, I_{N}\right), \\
\operatorname{det} \frac{\partial^{2} F}{\partial I_{j} \partial I_{k}^{*}} & \neq 0 .
\end{aligned}
$$

\section{Conclusions}

We have defined a new type of local parametric statistics of quantum eigenstates, which describes the statistical distributions of zeros of Husimi functions and the velocities the derivatives of zeros with respect to an external parameter. It has been conjectured 
that apart from the scaling parameters (density of zeros and mean square velocity) the parametric statistics of zeros of a Husimi representation of an eigestate of a classically chaotic system should behave universally and should be well described by the ensembles of Gaussian random polynomials to the same extent as the statistics of energy spectra, eigenvector components, or matrix elements are described by the ensembles of Gaussian random matrices. The parametric statistics of roots of Gaussian random polynomials (or zeros of Gaussian random holomorphic functions) have been solved analytically and its applicability to simple chaotic Hamiltonian systems has been demonstrated numerically.

In case where the dependence upon external parameter is linear the flow of zeros of an analytic (Bargmann) function has been formulated in terms of fully integrable dynamical system, where the external parameter and the zeros are playing the roles of fictitious time and mutually repelling quasi particles, respectively. The analytical results on local parametric statistics presented in the first part of this paper are nothing else but 'equal-time' correlation functions of such a dynamical system. The fact that the velocity distribution (35) is a generalized Lorentzian and not Maxwellian is due to nonseparability of kinetic and potential energy. For example, the 2-body case $(N=2)$ in the center of mass frame $v=v_{1}=-v_{2}=2 \dot{z}, z=z_{2}-z_{1}$ can be described by the Hamiltonian

$$
H=\frac{1}{2}|\dot{z}|^{2}|z|^{2}=\frac{1}{2}|p|^{2} /|z|^{2}
$$

where $p=|z|^{2} \dot{z}$ is a (complex) momentum conjugated to the (complex) coordinate $z$.

The generalization to the calculation of the most general 'different-time' correlation function - non-local parametric $k$-point correlation function

$$
\tilde{\rho}\left(\boldsymbol{z}^{\prime}, \boldsymbol{v}^{\prime}, \boldsymbol{\lambda}\right)=\left\langle\prod_{j=1}^{k} \delta\left(z_{j}^{\prime}-z_{j}\left(\lambda_{j}\right)\right) \delta\left(v_{j}^{\prime}-(d / d \lambda) z_{j}\left(\lambda_{j}\right)\right)\right\rangle
$$

is straightforward by putting

$$
f_{j}=f\left(z_{j}, \lambda_{j}\right), \quad f_{j}^{\prime}=\partial_{z} f\left(z_{j}, \lambda_{j}\right), \quad \tilde{f}_{j}=(d / d \lambda) f\left(z_{j}, \lambda_{j}\right)
$$

instead of (6, 7,8) and following the formalism of section 2.

\section{Acknowledgements}

The hospitality of the Institut Henri Poincaré, Paris, where the major part of this work has been done, and the financial support of C.I.E.S. (France) and the Ministry of Science and Technology of the Republic of Slovenia are gratefully acknowledged.

\section{References}

[1] Bargmann V 1961 Comm. Pure Appl. Mat. 14 187; 1967 Comm. Pure Appl. Mat. 201

[2] Bogomolny E B, Bohigas O and Leboeuf P 1992 Phys. Rev. Lett. 682726

[3] Bogomolny E B, Bohigas O and Leboeuf P 1995 "Quantum Chaotic Dynamics and Random Polynomials", preprint, IPN Orsay 
[4] HAAKE F "Quantum Signatures of Chaos" (Springer-Verlag Berlin 1991)

[5] Hannay J H 1995 J. Phys. A: Math. Gen. 29 L101

[6] Leboeuf P and Shukla P, 1995 preprint, IPN Orsay

[7] Leboeuf P And Voros A, in "Quantum Chaos" eds. G. Casati and B. V. Chirikov (Cambridge University Press, Cambridge 1993)

[8] Pechukas P 1983 Phys. Rev. Lett. 51943

[9] Prosen T 1995 J. Phys. A 28 L349

[10] Prosen T 1995 Physica D 91244

[11] Prosen T 1995 Numerical results on 2-point statistics of zeros of Husimi functions of extremely high lying eigenstates in a semiseparable oscillator, unpublished

[12] Prosen T 1996 "Exact statistics of complex zeros of Gaussian random polynomials with real coefficients", preprint CAMTP/96-2, submitted to J. Phys. A

[13] Yukawa T 1985 Phys. Rev. Lett. 541883

\section{Figure captions}

Figure 1: The numerical velocity distribution of the chaotic semiseparable oscillator (full curve) (see text for details) as compared to the theoretical velocity distribution (35) based on the theory of random polynomials (dashed curve). The agreement is highly statistically significant. 